\title{
Applied Systems Theory: Wind Turbine Output Power Prediction Based on Wind Energy Utilization Coefficient
}

\author{
Xiaotong Wang, Wangqiang Niu, Wei Gu \\ Key Laboratory of Transport Industry of Marine Technology and Control Engineering, Shanghai \\ Maritime University, Shanghai 201306, China
}

Received: September 14, 2020. Revised: March 21, 2021. Accepted: April 8, 2021. Published: April 12, 2021.

\begin{abstract}
The output power of a wind turbine is the most critical variable reflecting the operating status of the turbine. To improve the interpretability of the prediction model, a segmented output power method based on wind energy utilization coefficient is established. First, the wind energy conversion system of the wind turbine is given, and the SCADA data of a wind turbine is visually analyzed. Then it is proposed to separate the data into three groups according to different operating regions of wind turbines: the Maximum Power Point Tracking region, the rotator speed control region, and the power control region. In the Maximum Power Point Tracking region, wind energy utilization coefficient is found by a fitted cubic polynomial of the tip speed ratio. In the rotator speed control region, a modeling method for determining wind energy utilization coefficient through dynamic labels is designed. In the power control region, the output power is kept at the rated value. Finally, the 3 models are connected so that time-series data can be handled. The SCADA data of a 2.1MW wind turbine is used to verify the above models. The performance of these models is given in the form of Root Mean Square Error, indicating that the output power predicted by this method has good accuracy. The segmented output power model based on wind energy utilization coefficient can simulate the operation process of wind turbines, and has good accuracy and interpretability.
\end{abstract}

Keywords-Systems Theory, Applied Systems Theory, Wind turbine; Output power prediction; Wind energy utilization coefficient; Power generation performance.

\section{INTRODUCTION}

$\mathrm{D}$ UE to the increasing concern for the environment, wind power generation has rapidly developed in new energy power generation by under its advantages such as low cost, clean and renewable. With the increase in the number of wind turbines, wind power companies pay more attention to the operation and management of turbines that have been put into operation. The power generation performance of wind turbines indicates the level of economic benefits of wind power companies. The power generation performance of wind turbines is analyzed with monitoring and grasping the operating status, abnormal operation and early failures can be detected in time, thereby reducing the maintenance costs of the wind turbines.

Lots of work has been done on how to reflect the operating status and power generation performance of wind turbines. Reference [1] studied the influence of different air densities on the performance of wind turbine blades and used genetic algorithms to optimize the design of blades to improve the power generation performance. Reference [2] focused on the influence of wind turbine blade airfoil design on the output power and gave the blade design concept and correction method. Using intelligent algorithms to model SCADA data can detect the status of a wind turbine. Such data mining methods usually don't require analysis of the mechanism of the wind turbine itself, thus their interpretability needs to be improved. Reference [3] used neural network algorithms on historical operating data to establish a normal behavior model for predicting the temperature of generator bearings. This model can make online predictions on real-time operating data, and evaluate the health condition of the bearing based on the residual index between the predicted value and the actual value. Reference [4] adopted empirical mode decomposition to extract feature vectors from vibration signals and used fuzzy support vector machines to solve outlier problems. Reference [5] established the conditionally expected wind output equation based on the interval estimation of wind speed.

The wind turbine power curve is an important visual model reflecting the performance of the wind turbine. Reference [6] considered the influence of the ambient temperature and nearby obstacles on the output of wind turbines and established an adaptive neuro-fuzzy interference system model. By predicting 
the power generation, it realized the monitoring of the operating status of wind turbines. Reference [7] regarded the power curve as the evaluation standard for the performance of wind turbines. Based on the Bins method to draw the wind power curve, non-parametric interval estimation was used to obtain the uncertainty estimation interval of the wind power curve, which improved the reliability of the estimation results. The input variables considered by above methods usually include natural environmental factors and wind turbine state variables. Some methods of time series are also used to predict the performance state of wind turbines in the future. Reference [8] proposed a stochastic wind power generation model based on the autoregressive integrated moving average (ARIMA) process, taking into account the non-stationarity, physical limitations, and time correlation of wind power generation. Reference [9] analyzed the chaotic characteristics of the time series of wind turbine operating status parameters and used the phase space reconstruction method to establish a weighted first-order local prediction model of the wind turbine operating status.

The wind energy conversion system is one of the important links in the energy conversion process of wind turbines. The wind energy utilization coefficient $C_{p}$ reflects the wind energy utilization efficiency of the wind turbines. It is an important indicator of the power generation performance and might be introduced into the output power curve model to strengthening the model interpretability. Several attempts have been tried. Reference [10] established a physics-based prediction model for a $120 \mathrm{~mW}$ miniature horizontal axis wind turbine, studied the performance of the generator rotor, and obtained the approximate value of the power coefficient of the generator rotor. Reference [11] uses the least square method to fit the wind turbine data to a curved surface, and linearize the fitted curved surface to obtain a method for identifying the $C_{p}$ of the wind turbine. Reference [12] analyzed the influence of factors such as pitch angle, yaw angle, and tip speed ratio $\lambda$ on the power generation performance of wind turbines, and discussed that the $C_{p}$ can reflect the operating status of the wind turbine.

In this study, a segmented output power model based on $C_{p}$ is suggested. First, data exploration, data visualization, and group processing according to the different operating regions of the wind turbine. Then, a segmented output power model based on $C_{p}$ is established, a method to determine through dynamic labels is proposed, the segmentation model is connected to make it suitable for time-series data, and the model is tested with actual data. Finally, comparisons with other studies are described and conclusions are drawn. Our method might be combined with [18],[19], [20].

\section{MATERIALS AND METHOD}

\section{A. Wind Turbine Characteristic Model}

The power generation system of a wind turbine is mainly composed of rotator blades, a mechanical transmission system, and a power control system [13], as shown in Fig. 1.
The rotating blades of a wind turbine obtain energy from the moving air and transfer it to the generator to generate electricity through the mechanical drive unit. Aerodynamic equations are calculated as follows:

$$
P_{\mathrm{w}}=\frac{1}{2} \rho \pi R^{2} v^{3}
$$

where $P_{\mathrm{w}}$ is the total wind power corresponding to air kinetic energy, $\rho$ is the air density, $R$ is the blade radius, and $v$ is the wind speed. Ideally, the power a wind turbine obtains from wind energy can be expressed as:

$$
\begin{gathered}
P_{a}=C_{p}(\lambda, \beta) P_{w}=\frac{1}{2} C_{p}(\lambda, \beta) \rho \pi R^{2} v^{3} \\
\lambda=\frac{\omega R}{v}
\end{gathered}
$$

where $P_{a}$ is the actual output power, $\lambda$ is the tip speed ratio, which is the ratio of the linear velocity of the wind turbine blade tip to the wind speed. The parameter $C_{P}(\lambda, \beta)$ is the wind energy utilization coefficient, a nonlinear function of the tip speed ratio $\lambda$ and the pitch angle $\beta . \omega$ is the blade angular velocity.

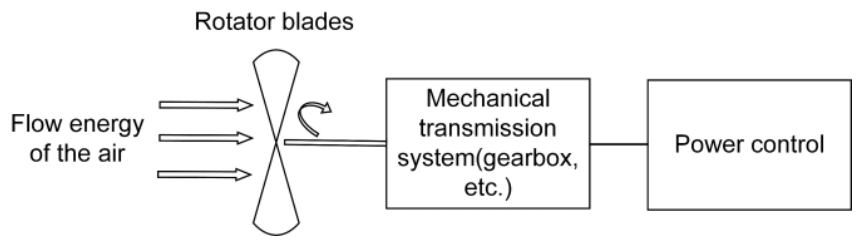

Fig. 1 Functional chain of a wind energy turbine The equation of $C_{p}(\lambda, \beta)$ obtained from [14] is:

$$
\begin{gathered}
C_{p}(\lambda, \beta)=\mathrm{C}_{1}\left(\frac{\mathrm{C}_{2}}{\lambda_{i}}-\mathrm{C}_{3} \beta-\mathrm{C}_{4} \beta^{\mathrm{C}_{5}}-\mathrm{C}_{6}\right) e^{-\frac{\mathrm{C}_{7}}{\lambda_{i}}} \\
\lambda_{i}=\frac{1}{\frac{1}{\lambda+\mathrm{C}_{8} \beta}-\frac{\mathrm{C}_{9}}{\beta^{3}+1}}
\end{gathered}
$$

According to different types of wind turbines, different parameters $\mathrm{C}_{1}-\mathrm{C}_{9}$ can be obtained, and the nonlinear relationship between $C_{p}(\lambda, \beta)$ and $\lambda$ and $\beta$ can be fitted. The maximum wind energy utilization coefficient $C_{p}$ does not exceed the Betz limit, which is 0.593 . The greater $C_{p}$, the more wind energy absorbed by the wind turbine, and the higher the power generation performance.

\section{B. Operating Regions Division of a Wind Turbine}

The rotator speed-power diagram of the variable speed variable pitch wind turbine [15] has been shown in Fig. 2, divided into different operating regions according to rotator speed. $0-n_{i n}$ is the grid-connected region, $n_{i n}-n_{r t d}$ is the Maximum Power Point Tracking (MPPT) region [16], the rotator speed control region is when the rotator speed 
reaches $n_{r t d}$ to the power reaches $P_{r t d}$, and the power control region is the speed greater than $n_{r t d}$.

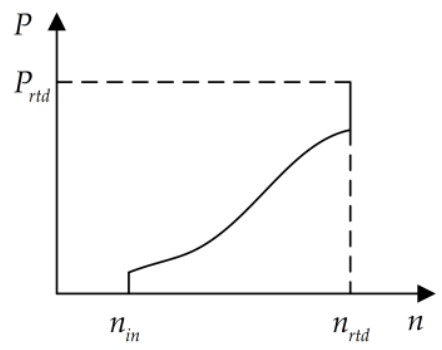

Fig. 2 Wind turbine operating regions

Among them, in the MPPT region, the pitch angle remains unchanged, ranging from $0^{\circ}$ to $2^{\circ}$. When the wind speed changes, the control system of the wind turbine adjusts the impeller speed in time by adjusting the electromagnetic torque. The tip speed ratio is maintained near the optimal value $\lambda_{\text {opt }}$ so that the wind turbine can obtain the maxi-mum $C_{p}$ and achieve higher power generation performance. When the wind speed reaches the rated value and the output power reaches the rated power, if the wind speed continues to increase, the pitch angle will increase to control the absorption of wind energy and ensure that the output power remains unchanged.

\section{Data Exploration}

The wind turbine to be studied is the variable speed variable pitch double-fed wind turbine with a rated power of 2.1 MW in a wind farm in Chongming, Shanghai, China. The cut-in wind speed, rated wind speed, and cut-out wind speed are $4 \mathrm{~m} / \mathrm{s}$, $11 \mathrm{~m} / \mathrm{s}$, and $25 \mathrm{~m} / \mathrm{s}$, respectively, and the impeller diameter is 105 $\mathrm{m}$. The SCADA data sampling time is 30 seconds, including variable information such as time, wind speed, output power, pitch angle, and yaw angle.

The $C_{p}$ is calculated by (1) and (2), where $P_{a}$ is the output power in the wind turbine data. The $\lambda$ is calculated by (3). The characteristic diagrams are shown in Fig. 3.

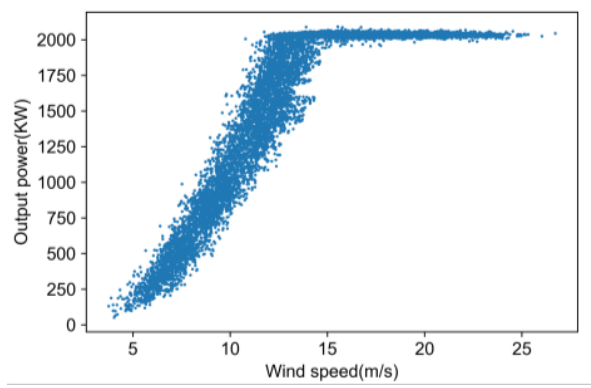

(a) wind speed-output power diagram

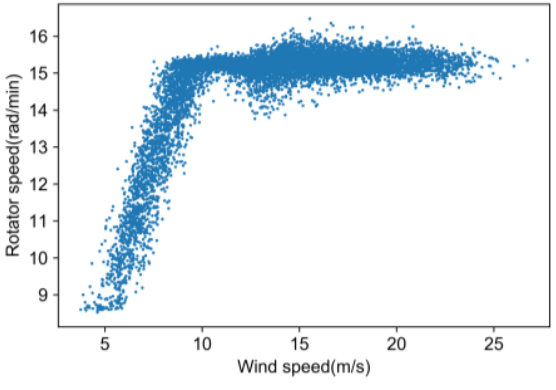

(b) wind speed-rotator speed diagram

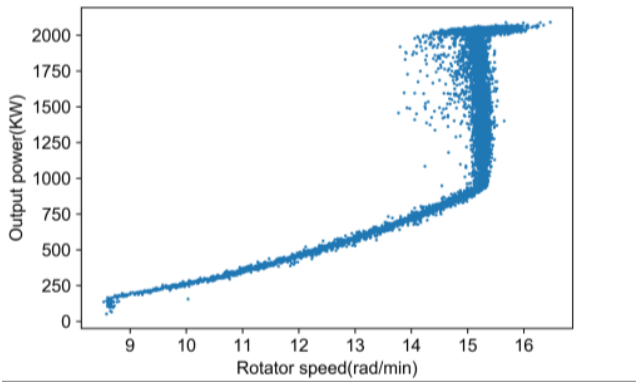

(c) rotator speed-output power diagram

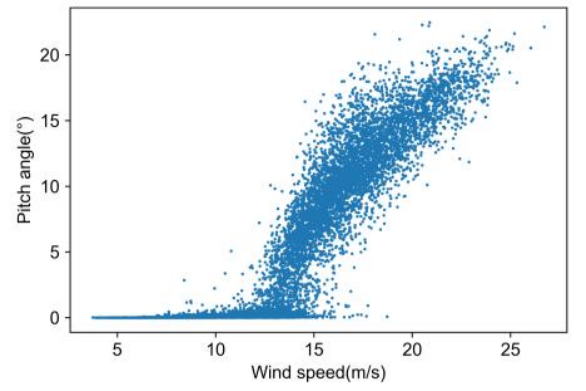

(d) wind speed-pitch angle diagram

Fig. 3 The characteristic diagrams of the wind turbine

The rated wind speed of this wind turbine is $11 \mathrm{~m} / \mathrm{s}$, as shown in Fig. 3(a). In Fig. 3(b), when the wind speed reaches $8.5 \mathrm{~m} / \mathrm{s}$, the rated rotator speed begins to appear. Combined with Fig. 3(c), the wind turbine is in the MPPT region from the start of the cut wind speed to $8.5 \mathrm{~m} / \mathrm{s}$. Fig. 3(b) shows that when the wind speed reaches $10 \mathrm{~m} / \mathrm{s}$, the rotator speed reaches around the rated speed, and the output power rises steadily. When the wind speed reaches $14 \mathrm{~m} / \mathrm{s}$, the output power is near the rated value, and this period is in the rotator speed control region. According to Fig. $3(d)$, the pitch angle is near 0 before reaching the rated wind speed, and the pitch angle starts to increase after the rated wind speed. When the wind speed is greater than $14 \mathrm{~m} / \mathrm{s}$, the output power of the wind turbine is rated power, and the wind turbine is in the power control region. At this time, the pitch angle needs to get rid of excess wind energy to prevent excessive output power.

Based on the above analysis, this study will group the data into 3 different operating regions: the MPPT region, the rotator speed control region, and the power control region.

1) The MPPT region. When the wind speed is less than $8.5 \mathrm{~m} / \mathrm{s}$, as shown in Fig. 4, the corresponding relationship between $\lambda$ and $C_{p}$ is all located on the right side of the 
image, showing an obvious functional relationship. At this time, the wind turbine is in the MPPT region. By adjusting the blade speed, the wind turbine is always near the best $\lambda$, to fully capture the wind energy.

2) The rotator speed control region. When the wind speed is greater than $8.5 \mathrm{~m} / \mathrm{s}$ and less than $14 \mathrm{~m} / \mathrm{s}$, the relationship between $\lambda$ and $C_{p}$ is shown in Fig. 5, which is located on the left side of the $\lambda-C_{p}$ diagram, showing a non-linear band-like distribution.

3) The power control region. For wind speed is greater than $14 \mathrm{~m} / \mathrm{s}$, the wind turbine must be in the power control region, so it is considered that the output power of the wind turbine under normal conditions is the rated power. This study focuses on modeling the first two operating regions.

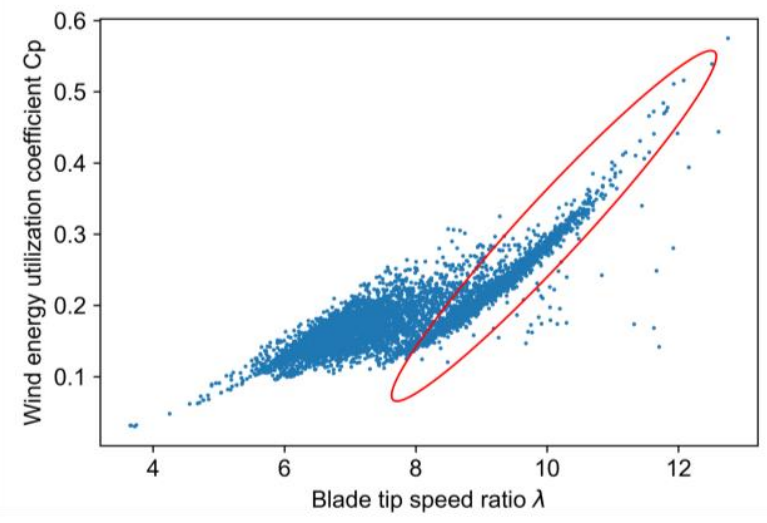

Fig. 4 Blade tip speed ratio $\lambda$ - wind energy utilization coefficient $C_{p}$ diagram in the MPPT

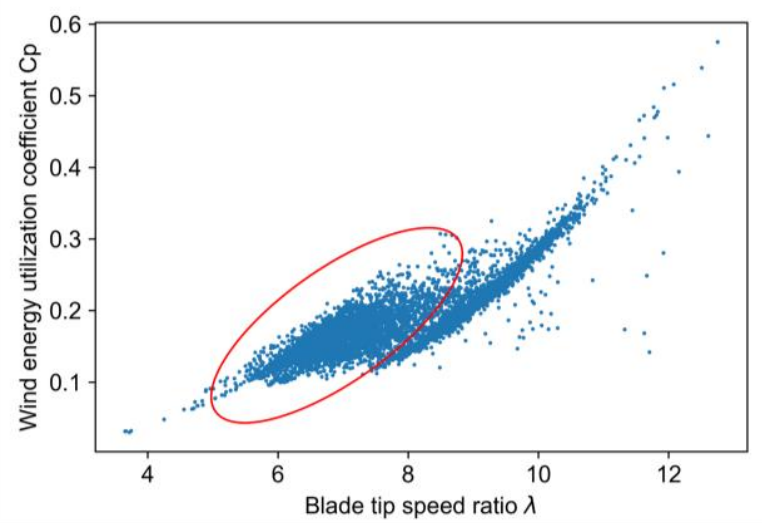

Fig. 5 Blade tip speed ratio $\lambda$ - wind energy utilization coefficient $C_{p}$ diagram in the rotator speed control region

\section{Model Based on Dynamic Label}

When the wind turbine operates in the rotator speed control region, it can be derived from formulas (2) and (3):

$$
C_{p} \square \frac{\lambda^{3} P_{a}}{n^{3}}
$$

At this time, the wind speed is greater than $8.5 \mathrm{~m} / \mathrm{s}$, and the rotator speed has reached near the rated speed, and it is reasonable to suppose that the rotator speed is constant.
Equation (6) shows that $C_{p}$ is proportional to $\lambda$ and the output power of the wind turbine. The data of the rotator speed control region is divided into 11 groups according to different ranges of output power. The relationship between $\lambda$ and $C_{p}$ is shown in Fig. 6.

As shown in Fig. 6, when grouped according to different power ranges, $\lambda$ and $C_{p}$ of each group of data shows a certain linear relationship, and this relationship can be fitted with simple mathematical methods. It is desired to determine a unique $C_{p}$ through $\lambda$. However, it can be seen from Fig. 5 that each $\lambda$ corresponds to multiple different wind energy utilization coefficients. Inspired by Fig. 6 , it is proposed to introduce a new variable label $_{i}$ to name the above 11 sets of data, as shown in (7).

$$
\text { label }_{i}=i, i=0,1, \ldots, 10
$$

The 11 labels represent the 11 corresponding relation-ships between $\lambda$ and $C_{p}$. label $_{i}$ is used as a new variable of the data to participate in the model establishment to determine $C_{p}$. Through the dynamic adjustment of labels, the purpose of predicting a unique $C_{p}$ is achieved.

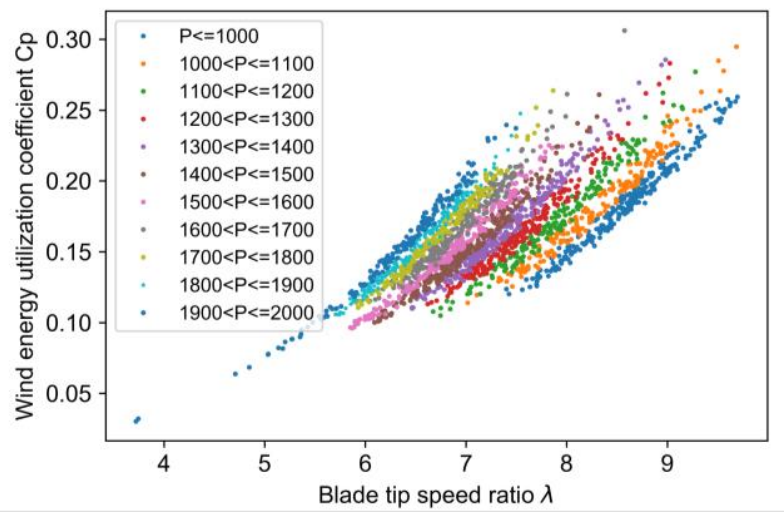

Fig. 6 The relationship between the tip speed ratio $\lambda$ and the $C_{p}$ in different power ranges

This paper proposes a dynamic label mechanism for determining the relationship between $\lambda$ and $C_{p}$ in the rotator speed control region, and the steps are as follows:

1) The above has completed grouping the data according to different power ranges, and the data has obtained new variables label $_{i}$.

2) This part of the modeling data is uniformly calculated for the tip speed ratio time series difference $\Delta \lambda=\lambda_{m}-\lambda_{m-1}$ and the label time series difference $\Delta$ label $=$ label $_{m}-$ label $_{m-1}$, where $\lambda_{m}$ is the tip speed ratio of the wind turbine at the $m$ th time point and label $_{m}$ is the wind turbine at the $m$ th time point time label.

3) According to the variable label $_{i}$, take out the current moment data corresponding to the data with the same label at the previous moment and determine the relationship 
between $\Delta \lambda_{i}$ and $\Delta l a b e l_{i}$ in this part of the data. This step is to study how much $\Delta$ label $_{i}$ will change in the next moment according to $\Delta \lambda_{i}$ when the data is on a certain label at the previous moment.

4) According to the relationship in step 3), get $\Delta$ label $_{t}=\Delta$ label $_{i}$, label $_{t-1}=$ label $_{i}$ at the previous moment.

The calculation method of the label at the current time $t$ is: label $_{t}=$ label $_{t-1}+\Delta$ label $_{t}$.

5) Calculate $C_{p}$ according to the fitting equation at each label.

Now the method of step 3) above will be described. The steps are as follows:

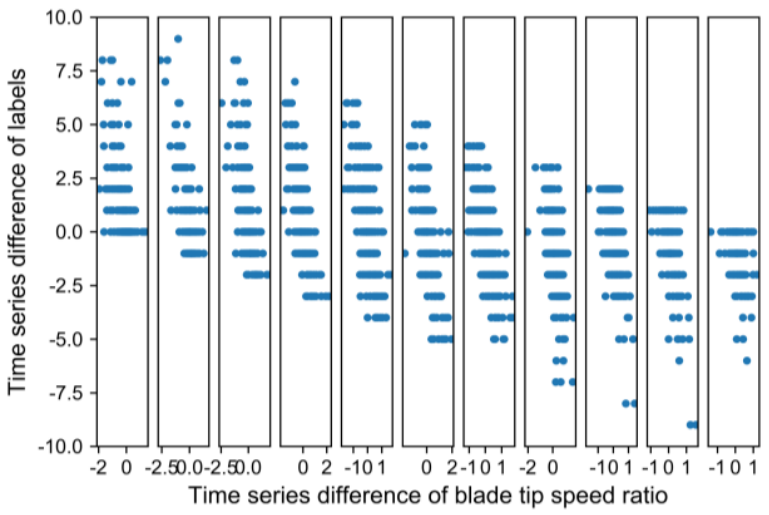

Fig. 7 The corresponding relationship between $\Delta \lambda_{i}$ and $\Delta$ label $_{i}$ under different labels

1) Determine the numerical relationship $\Delta \lambda_{i}$ and $\Delta$ label $_{i}$. Take out the data, which have the same label at the previous moment, and observe the change of $\Delta$ label $_{i}$ with $\Delta \lambda_{i}$, as shown in Fig.7. Fig. 7 shows the data at the current moment which is on the same label at the previous moment. The overall relationship between $\Delta \lambda_{i}$ and $\Delta$ label $_{i}$ is inversely proportional, and the distribution is scattered. Since the least-squares fitting is susceptible to outliers, it will cause a greater error, so it cannot be used for curve fitting. Therefore, this article proposes the following processing methods. According to the variation range of $\Delta \lambda_{i}$ between -3 and 2 , divide $\Delta \lambda_{i}$ into 25 intervals with 0.2 as the step, count the probability value of $\Delta$ label $_{t}$ corresponding to $\Delta \lambda_{i}$ in each interval, and then take the $\Delta l a b e l_{t}$ with the highest probability in each interval as the final value of $\Delta \lambda_{i}$. The essence of this step is to deter-mine the numerical relationship between $\Delta \lambda_{t}$ and $\Delta$ label $_{t}$, and predict the next moment label.

2) Consider the influence of continuous-time and modify the numerical relationship. Since the wind turbine is a complex nonlinear coupling system with inertia, the state at the current moment is related to the state at the previous moment. Even if the wind speed and rotation speed are the same at two moments, the corresponding output power may be different. The ordinary wind speed-output power, wind speed-rotation speed, and other characteristic curves lose time information, and it is difficult to reflect the inertia effect of wind turbines. Therefore, this paper also considers the influence of continuous-time. Put the numerical relationship in the continuous-time data and correct the obtained numerical relationship. The rules are as follows: Arrange the data in chronological order. If $\Delta \lambda$ continuously rises and slabel calculated from the numerical value is 0 , the predicted value of the label will remain unchanged for a while. At this time, $\Delta$ label needs to be adjusted to decrease; similarly, if $\Delta \lambda$ continuously falls, the $\Delta$ label obtained by numerical calculation is 0 , so that the predicted value of the label remains unchanged for a while. At this time, $\Delta$ label needs to be adjusted to increase. This is because the numerical result of $\Delta$ label $_{i}$ is obtained by selecting the maximum value of $\Delta$ label $_{i}$ in each interval, and there is a certain error. If the $\lambda$ continues to increase or decrease within a period of time, the output power will definitely change, and the corresponding label must also change. Therefore, adjustments should be made to the results that remain unchanged.

To sum up, firstly calculate the single-step change, and then put the single-step prediction result into continuous time. According to the data change pattern, if the adjustment condition is met, $\Delta$ label $_{i}$ is corrected to obtain the final result $\Delta$ label $_{\text {opt }}$. The method in the rotator speed control region is shown in Fig. 8.

Since the data above is divided according to the operating regions, each model is only suitable for the data under one operating region, so the following processing needs to be done in the connection and use of the model:

1) The model of the MPPT region is a cubic curve obtained by the fitting. Regardless of the state at the previous moment, as long as the wind speed condition is met, the model can be used for calculation.

2) In the power control region, as long as the wind speed and speed conditions are met, the output power of the wind turbine is considered to be the rated power.

3) The rotator speed control region is in the middle position in the 3 operating regions. At this time, the control state of each system of the wind turbine is complicated. When the front and back two operating regions are switched to this region, the initial state of the model in this region will be changed. Therefore, set the data label of the MPPT region to be label $_{i}=0$, and set the data label of the power control region to be label $_{i}=10$ to connect the three regions.

The main idea to predict the output power of wind turbines is summarized in Fig. 9. Input continuous SCADA data and divide the data into three groups according to different wind speed ranges, corresponding to three operating regions of wind turbines. In the first two regions, $C_{p}$ is obtained by 
calculating $\lambda$ or labels, then $P$ is calculated. Finally, three regions are connected by the connection criterion.

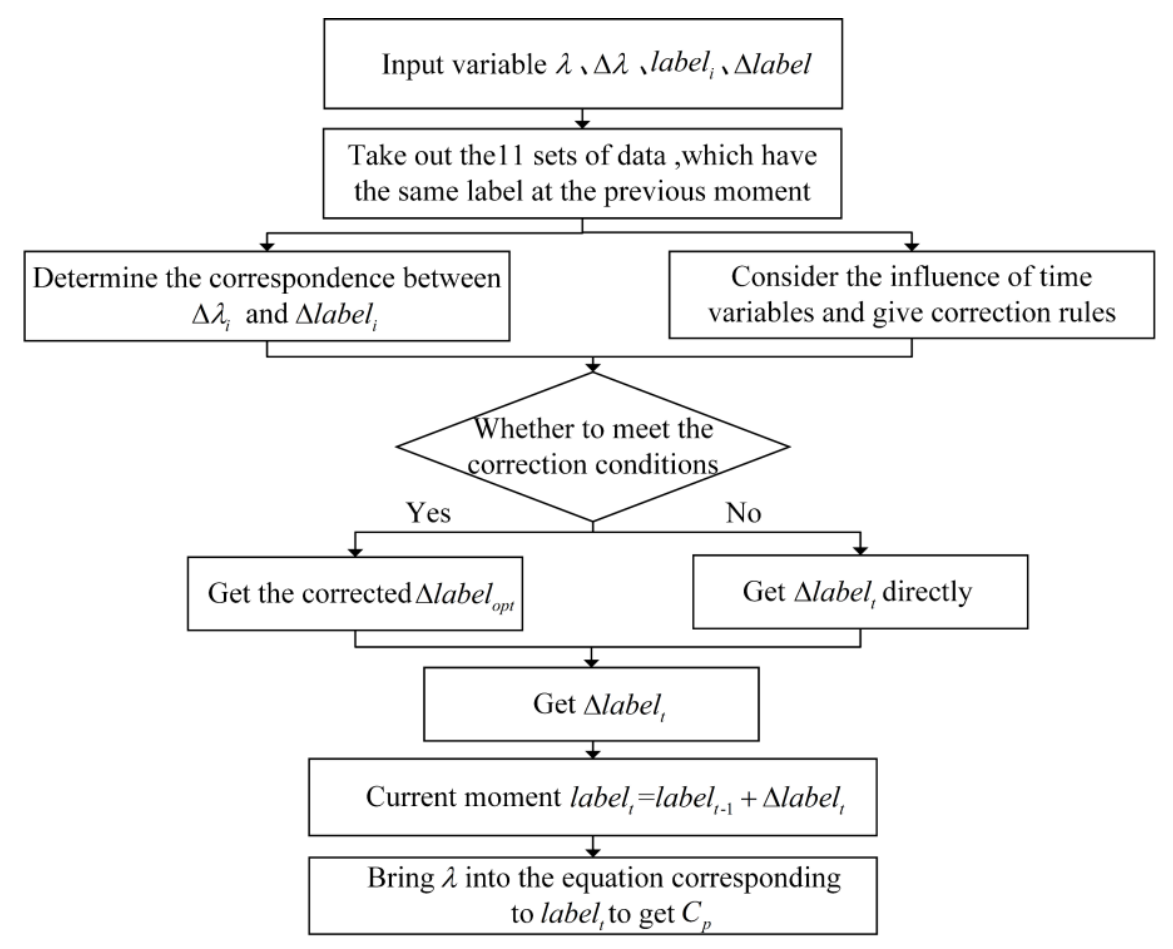

Fig. 8 Flow chart of calculation of wind energy utilization coefficient $C_{p}$ in the rotator speed control region

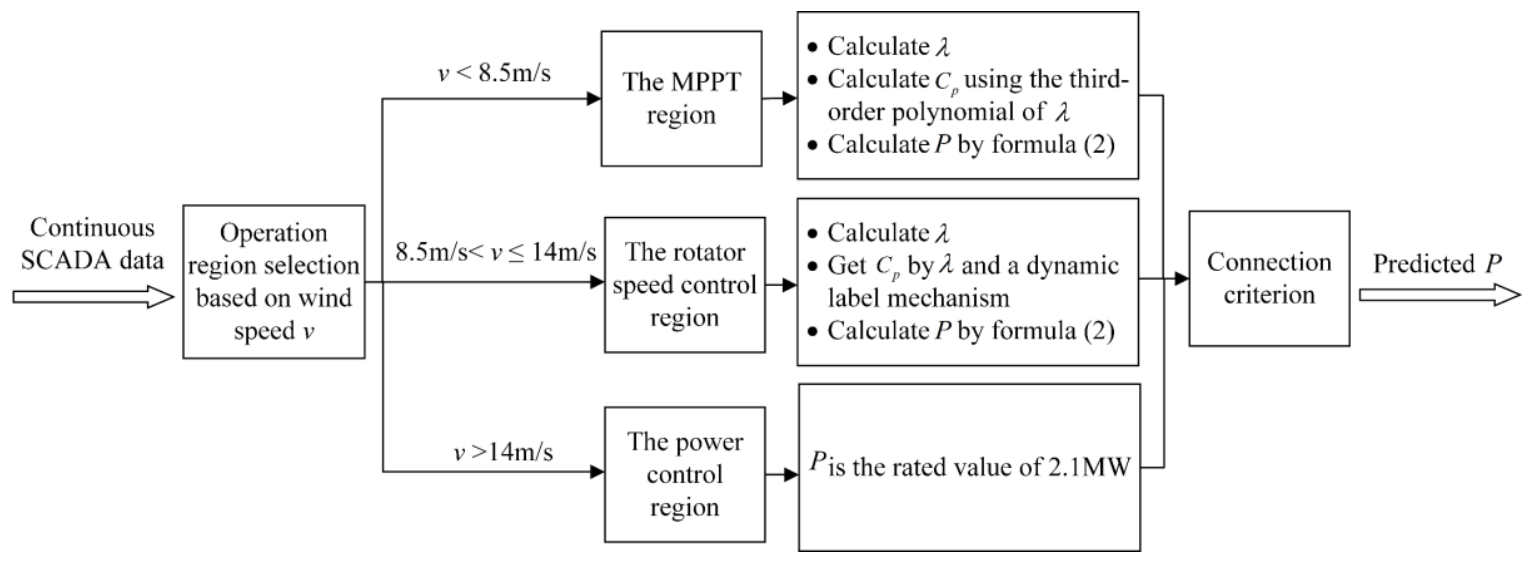

Fig. 9 The summarized model for predicting output power of wind turbines

\section{RESULTS}

\section{A. Data Experiment in the MPPT Region}

Select 997 pieces of eligible continuous-time data as training data. At this time, the wind speed is between $4-8.5 \mathrm{~m} / \mathrm{s}$. The $\lambda$ and $C_{p}$ present a concentrated nonlinear relationship, as shown in Fig. 10. Using SPSS software and the least square method to fit the data, a cubic curve (8) with a good fitting effect can be obtained in Fig. 10.

The fitted $C_{p}$ is:

$$
C_{p}=0.00516-0.000378 \lambda^{2}+0.000318 \lambda^{3}
$$

In the MPPT region, the goal of the control strategy of the wind turbine main control system is to maintain the wind turbine near the best $\lambda$. The adjustment and operating process of the wind turbine is a dynamic change process, and the polynomial model (8) obtained by fitting conforms to the operation law of wind turbines. 


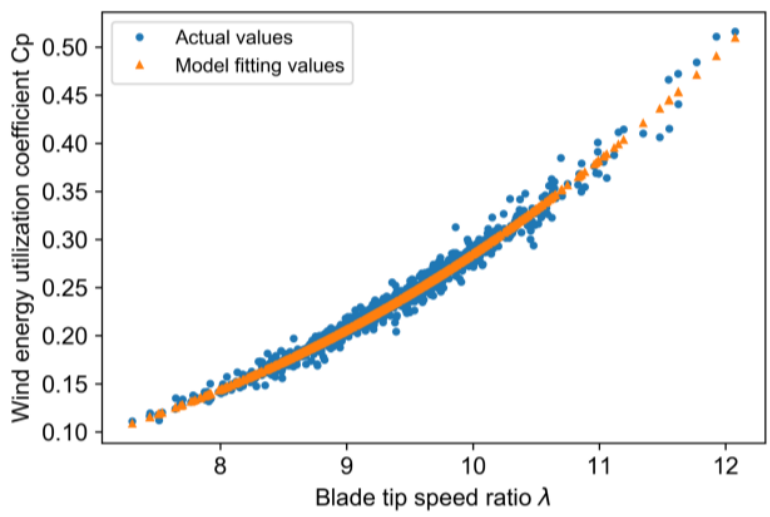

Fig. 10 The fitting curve of $C_{p}$ of the training data

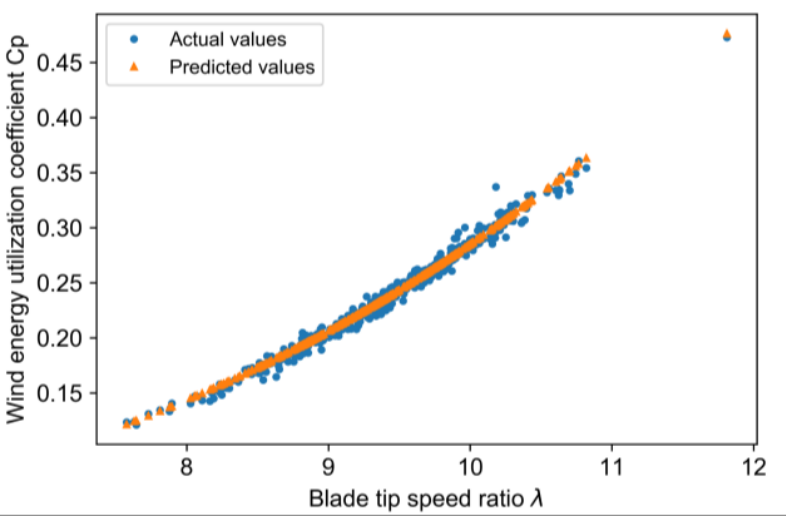

Fig. 11 The actual and predicted $C_{p}$ of test data with $\lambda$

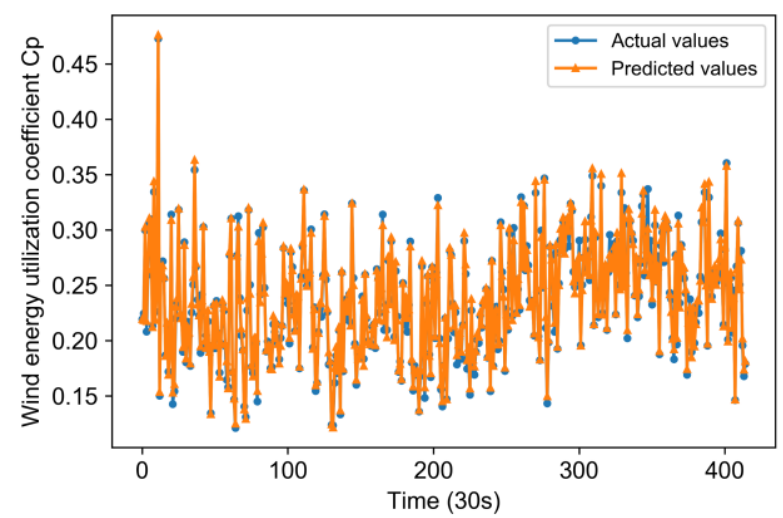

Select 390 pieces of continuous-time data that meet the conditions as the test data to test the performance of the fitted cubic model. The prediction of the relationship between $\lambda$ of the test data and $C_{p}$ is shown in Fig. 11. The prediction of $C_{p}$ that changes continuously with time is shown in Fig. 12.

From the wind power generation model (2), the output power of the wind turbine over time can be predicted in Fig. 13. It can be seen from Fig. 11, 12, and 13 that the cubic model between $\lambda$ and $C_{p}$ shows higher prediction accuracy on the test data. Compared with the actual value of $C_{p}$, the root mean square error (RMSE) of the residual between the actual value and the predicted value is 0.0058 .

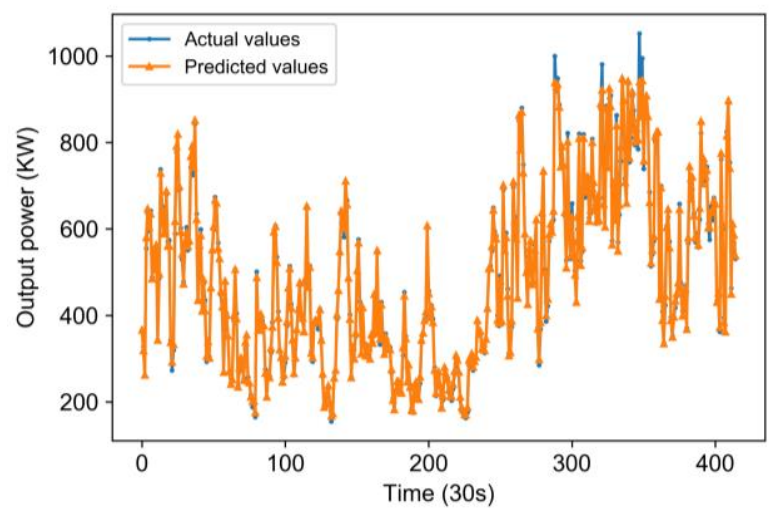

Fig. 13 Power prediction results of test data

\section{B. Data Experiment in the Rotator Speed Control Region}

From the analysis of Section II, this part of the data includes 3010 pieces of data whose wind speed is greater than $8.5 \mathrm{~m} / \mathrm{s}$ and does not reach the rated power. The relationship between $\lambda$ and $C_{p}$ presents a band-like nonlinear distribution, as shown in Fig. 5. It is difficult to fit through functions. Therefore, new variables label $_{i}$ are set according to different power ranges, and the defined standards are shown in Table 1.

Fig. 12 The actual and predicted $C_{p}$ of test data with time

Table 1 Labels definition based on power ranges

\begin{tabular}{c|c|c|c|c|c|c|c|c|c|c|c}
\hline $\begin{array}{c}\text { Power range } \\
(\mathrm{KW})\end{array}$ & $<=\mathbf{1 0 0 0}$ & $\begin{array}{c}\mathbf{1 0 0 0}- \\
\mathbf{1 1 0 0}\end{array}$ & $\begin{array}{c}\mathbf{1 1 0 0}- \\
\mathbf{1 2 0 0}\end{array}$ & $\begin{array}{c}\mathbf{1 2 0 0}- \\
\mathbf{1 3 0 0}\end{array}$ & $\begin{array}{c}\mathbf{1 3 0 0}- \\
\mathbf{1 4 0 0}\end{array}$ & $\begin{array}{c}\mathbf{1 4 0 0}- \\
\mathbf{1 5 0 0}\end{array}$ & $\begin{array}{c}\mathbf{1 5 0 0}-\mathbf{1 6 0 0} \\
\mathbf{1 6 0 0} \\
\mathbf{1 7 0 0}\end{array}$ & $\begin{array}{c}\mathbf{1 7 0 0}- \\
\mathbf{1 8 0 0}\end{array}$ & $\begin{array}{c}\mathbf{1 8 0 0}- \\
\mathbf{1 9 0 0}\end{array}$ & $>\mathbf{1 9 0 0}$ \\
\hline label $_{i}$ & label $_{0}$ & label $_{1}$ & label $_{2}$ & label $_{3}$ & label $_{4}$ & label $_{5}$ & label $_{6}$ & label $_{7}$ & label $_{8}$ & label $_{9}$ & label $_{10}$ \\
\hline
\end{tabular}

Table $2 \lambda-C_{p}$ fitting equations under each label

\begin{tabular}{c|c}
\hline Power range & $\lambda-C_{p}$ fitting equation \\
\hline$<=1000$ & $C_{p}=0.684-1.1029 \lambda+0.699 \lambda^{2}-0.114 \lambda^{3}$ \\
\hline
\end{tabular}




\begin{tabular}{c|c}
\hline $1000-1100$ & $C_{p}=\exp (-4.605+0.348 \lambda)$ \\
\hline $1100-1200$ & $C_{p}=\exp (-4.658+0.366 \lambda)$ \\
\hline $1200-1300$ & $C_{p}=\exp (-4.663+0.377 \lambda)$ \\
\hline $1300-1400$ & $C_{p}=\exp (-4.643+0.384 \lambda)$ \\
\hline $1400-1500$ & $C_{p}=\exp (-4.804+0.415 \lambda)$ \\
\hline $1500-1600$ & $C_{p}=\exp (-4.817+0.426 \lambda)$ \\
\hline $1600-1700$ & $C_{p}=\exp (-4.666+0.414 \lambda)$ \\
\hline $1700-1800$ & $C_{p}=\exp (-4.742+0.433 \lambda)$ \\
\hline $1800-1900$ & $C_{p}=\exp (-4.805+0.451 \lambda)$ \\
\hline$>1900$ & $C_{p}=\exp (-5.118+0.508 \lambda)$ \\
\hline
\end{tabular}

The 11 labels represent 11 relationships between the tip speed ratio $\lambda$ and the $C_{p}$. The 11 labels convert the band-like distribution into a line-like distribution and facilitate curve fitting. The SPSS software is used to fit 11 sets of data to obtain the corresponding fitting curves, as shown in Table 2.

751 pieces of new test data is used to verify the modeling method.

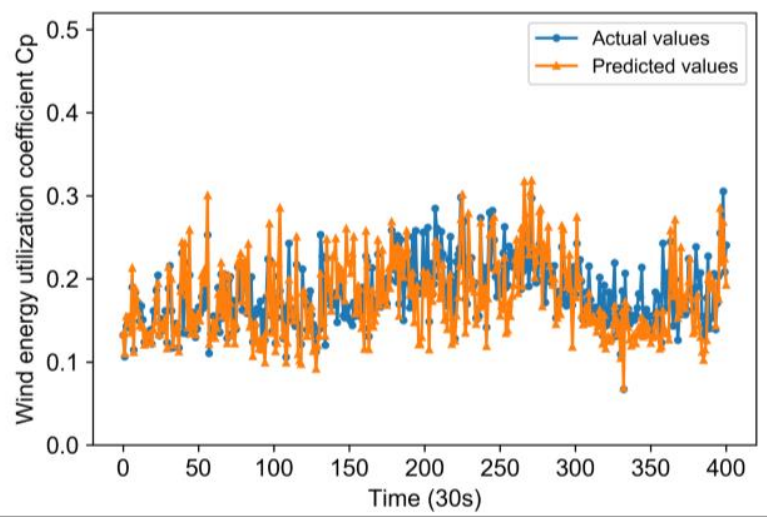

Fig. 14 Comparison of actual and predicted values of wind energy utilization coefficient from test data

In Fig. 14, the changing trend of the predicted $C_{p}$ is similar to the actual value. Where the test data changes sharply, the predicted $C_{p}$ shows a gentle change with a root mean square error (RMSE) of 0.029, which has high accuracy. According to (2), the output power of the wind turbine is calculated. As shown in Fig. 15, the predicted output power has the same changing trend as the real value.

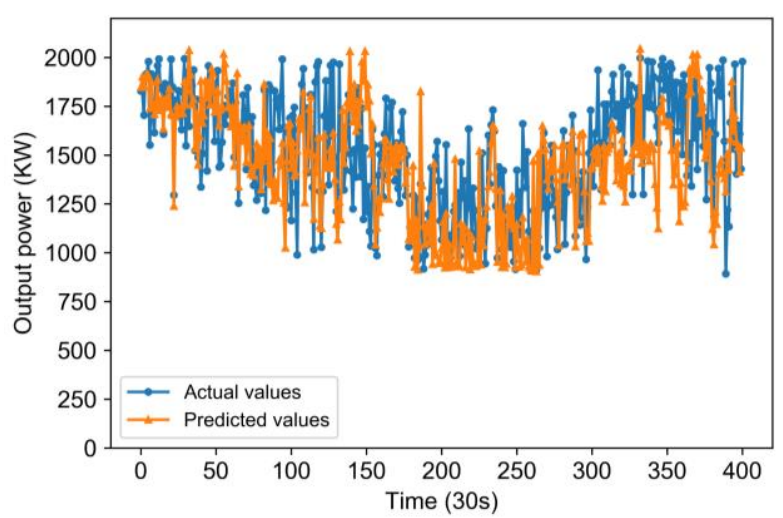

Fig. 15 Comparison of actual and predicted output power of test data

\section{A Comprehensive Experiment of Continuous Time-series} Data

Take 200 pieces of continuous data for a certain period as the test data. The data cover all the 3 operating regions, the MPPT region, the rotator speed control region, and the power control region. These data are used to verify the connected models established in Section 2.5. The prediction results of $C_{p}$ and output power are shown in Fig. 16 and Fig. 17.

The predicted value has a similar trend to the true value. Where the actual value changes quickly, the model predicted value changes more slowly. The RMSE of the predicted value of $C_{p}$ is 0.027 , which has high accuracy.

The wind speed-output power relationship diagram of the model is shown in Fig. 18. Compared with the power curve by the Bins method in [7], the model in this paper does not give the point value of the output power at a certain wind speed.

The wind speed-output power scatter points in this study present a loose strip-like distribution, which can more effectively reflect the relationship between wind speed and output power of wind turbines. 


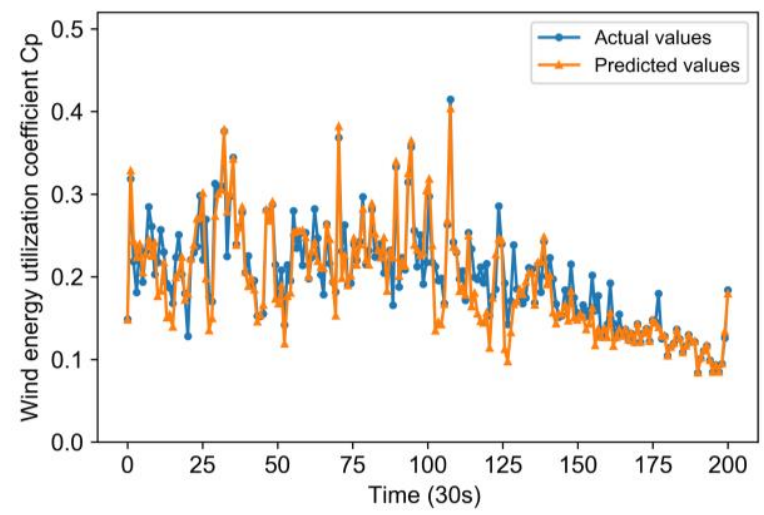

Fig. 16 Comparison of actual and the predicted values of wind energy utilization coefficient $C_{p}$

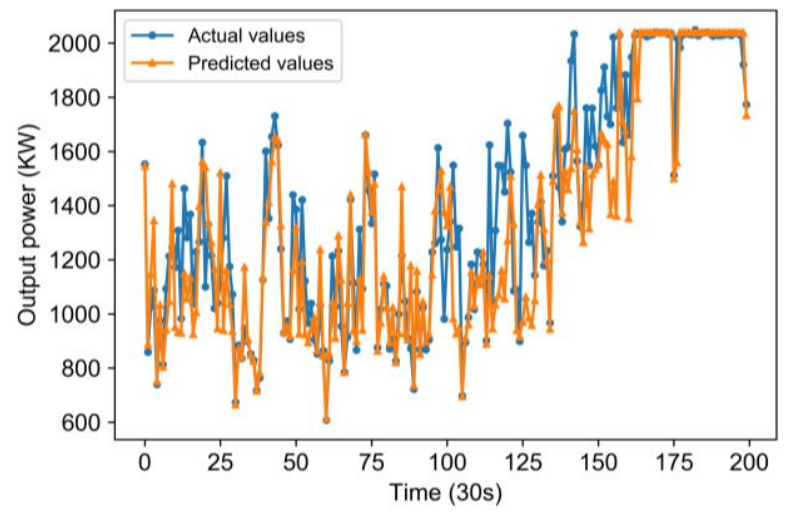

Fig. 17 Comparison of actual value and the predicted value of output power from test data

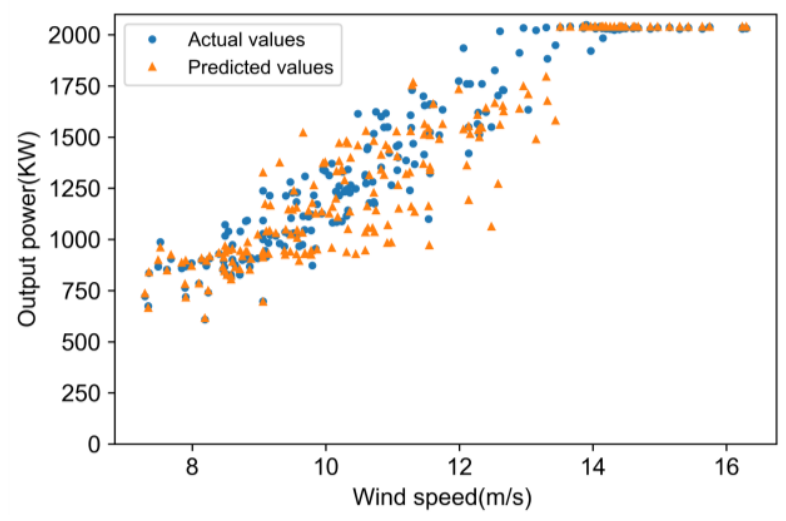

Fig. 18 Wind speed-output power scatter plot of test data

\section{Comparison and Discussion}

In the MPPT region, the least square method is used to directly fit the curve of $\lambda$ and $C_{p}$. In the rotator speed control region, the intermediate variable label is introduced to solve the problem of one $\lambda$ corresponds to multiple $C_{p}$. SPSS is used to fit the relationship between $\lambda$ and $C_{p}$ on each label. In the rotator speed control region, to determine the relationship between the tip speed ratio time series difference and the label time series difference, this paper takes the maximum probability of the tip speed ratio time series difference interval. The influence of the inertia of the wind turbine is taken into account to optimize the results. Determine the $C_{p}$ in which label should be taken at the current moment, and calculate the power generation based on the model of the wind turbine power generation. In the power control region, it is considered that the wind turbine generator reaches the rated power. After building the models, the connected model is given. In the part of model verification, first, the two models are verified with two types of data that meet the model input conditions, and then the connected model is verified with continuous-time data covering the entire region. Experiments show that the $C_{p}$ fitted by this method has good accuracy.

At the same time, this method can simulate the operating process of wind turbines. Compared with intelligent algorithms, the model has better interpretability. Compared with the use of Gaussian process regression to establish the output power model in [12], the model established in this article is equivalent to the white-box model. Although accuracy is sacrificed to a certain extent, the knowledge of the wind turbine mechanism is used as the theoretical support. The modeling method is easy to understand. In addition, the common modeling methods using intelligent algorithms, generally do not consider the time sequence, while the method in this paper considers the impact of the state of the wind turbine at the previous moment on the current state of the wind turbine, reflecting the operating process of the wind turbine.

Compared with the sixth-order polynomial wind power curve model [17], it only considered the single variable wind speed and didn't consider the influence of other variables on the model. The relationship between the wind speed and the output power presented was a one-to-one correspondence. In this paper, the wind speed, rotator speed, and tip speed ratio are used for modeling. The relationship between wind speed and output power is distributed in a band, which is closer to the real situation.

\section{CONCLUSION}

Starting from the wind energy conversion system of the wind turbine, this article visually analyzes the variables of the SCADA data. According to the different operating regions of the wind turbine, the data is grouped and processed, and three models of the relationship between $\lambda$ and $C_{p}$ are established. Experiments show that the $C_{p}$ fitted by this method has good accuracy, and verifies the feasibility of the proposed method in predicting output power.

The output power is a direct reflection of the power generation performance of the wind turbines. The prediction results obtained from the modeling can be used to monitor the operating status of the wind turbines and discover abnormal operation and early failure in time. For example, the $3 \sigma$ principle may be used for the residual difference between the predicted value and the true value to determine whether the state of the wind turbine is normal. This will be the direction of future work. 


\section{ACKNOWLEDGMENT}

The authors would like to acknowledge insight discussions with Professor Xixia Huang, from Shanghai Maritime University for this work.

\section{References}

[1] P. Abolfazl, M. Masoud, E. Reza and W. David, "Effect of air density on the performance of a small wind turbine blade: A case study in Iran", Journal of Wind Engineering and Industrial Aerodynamics, vol. 126, no. 2, pp. 1-10, 2014.

[2] H. Yu and H. Sun, "Airfoil Design and Aerodynamic Performance Analysis of Wind Turbine Blade", Materials Reports, vol. 34, pp. 466-468, 2020 (in Chinese).

[3] A. Kusiak, X. Wei, A. P. Verma and E. Roz, "Modeling and Prediction of Rainfall Using Radar Reflectivity Data: A Data-Mining Approach", IEEE Transactions on Geoscience and Remote Sensing, vol. 51, no. 4, pp. 2337-2342, 2013.

[4] J. Hang, J. Zhang, and M. Cheng, "Application of multi-class fuzzy support vector machine classifier for fault diagnosis of wind turbine," Fuzzy Sets \& Systems, vol. 297, pp. 128-140, 2016.

[5] H. Liu, J. Shi, and E. Erdem, "An Integrated Wind Power Forecasting Methodology: Interval Estimation of Wind Speed, Operation Probability of Wind Turbine, and Conditional Expected Wind Power Output of A Wind Farm," International Journal of Green Energy, vol. 10, pp. 151-176, 2013.

[6] S. Meik, I.F. Santos and S. Achiche, "Using Data-Mining Approaches for Wind Turbine Power Curve Monitoring: A Comparative Study". IEEE Transactions on Sustainable Energy, vol. 4, pp. 671-679, 2013.

[7] P. Lin, S. Zhao, Y. Xie, and Y. Hu, "Wind power curve modeling based on measured data and uncertainty estimation". Electric Power Automation Equipment, vol. 35, no. 004, pp. 90-95, 2015.

[8] P. Chen, T. Pedersen, B. Bak-Jensen, and Z. Chen, "ARIMA-Based Time Series Model of Stochastic Wind Power Generation," IEEE Transactions on Power Systems, vol. 25, no. 2, pp. 667-676, 2010.

[9] X. An; D. Jiang, "Chaotic characteristics identification and trend prediction of running state for wind turbine". Electric Power Automation Equipment, vol. 30, pp. 15-19, 2010 (in Chinese).

[10] F. Xu, F.G. Yuan, L. Liu, J. Hu, and Y. Qiu. "Performance Prediction and Demonstration of a Miniature Horizontal Axis Wind Turbine". Journal of Energy Engineering, vol. 139, no. 3, pp.143-152 2013.

[11] J. Wang, S. Lu, X. He, and J. Xia, "Study on parameters matching of rotator power coefficient for large scale wind turbine". Acta Energiae Solaris Sinica, vol. 33, pp. 221-225, 2012 (in Chinese).

[12] P. Guo, M. Jiang, and H. Li. "Performance analysis and monitoring based on SCADA data and Gaussian process regression for wind turbine power generation". Electric
Power Automation Equipment, vol. 36, no. 008, pp. 10-15,25, 2016. (in Chinese).

[13] S. Heier, "Grid integration of wind energy: onshore and offshore conversion systems, 3rd ed.”, John Wiley \& Sons: Chichester, U.K., pp. 1-3, 2014.

[14] J. Hang, J. Zhang, and M. Cheng, "Application of multi-class fuzzy support vector machine classifier for fault diagnosis of wind turbine," Fuzzy Sets \& Systems, vol. 297, pp. 128-140, 2016.

[15] Q. Sun, C. Liu, and C. Zhen, "Abnormal Detection of Wind Turbine Operating Conditions Based on State Curves". Journal of Energy Engineering, vol. 145, no. 5, pp.145, 2019.

[16] R. Saravanakumar and D. Jena, "Nonlinear control of a wind turbine based on nonlinear estimation techniques for maximum power extraction," International journal of green energy, vol. 13, no. 1-5, pp. 309-319, 2016.

[17] M. Marciukaitis, I. Zutautaite, L. Martisauskas, B. Joksas, G. Gecevicius, and A. Sfetsos, "Non-linear regression model for wind turbine power curve," Renewable Energy, vol. 113, pp. 732-741, 2017.

[18]Kuan-Wei Wu, Mansour Karkoub, Tzu-Sung Wu "Robust Adaptive Fuzzy Control Design for 3-D Tower Crane with Time Delayed States", pp.26-33, Volume 2, 2020, International Journal of Electrical Engineering and Computer Science (EEACS),

[19]D. Barth, I. A. Gorlach, G. Gruhler, "Modelling of a Thermal Spraying Controller Using MATLAB/Simulink", pp.79-84, Volume 2, 2020, International Journal of Electrical Engineering and Computer Science (EEACS)

[20] Pardeep Kumar, Hari Mohan, G. A. Hoshoudy, "FLR Effect on Stability of a Plasma in Porous Medium" pp.90-97, Volume 2, 2020, International Journal of Electrical Engineering and Computer Science (EEACS),

Xiaotong Wang received the B.E. degree from Shandong Agricultural University, Taian, China, in 2018.

She is currently pursuing the M.E. degree with Shanghai Maritime University, Shanghai, China. Her research interests include data mining and data analysis.

Wangqiang Niu received the B.E. degree from Xi'an Aerotechnical College, Xi'an, China, in 1998, the M.E. degree from Northwestern Polytechnical University, Xi'an, in 2004, and the Ph.D. degree from Shanghai Jiao Tong University, Shanghai, China, in 2008.

From 2013 to 2014, he was a Visiting Lecturer with McMaster University, Hamilton, ON, Canada. Since 2008, he has been a Lecturer with Shanghai Maritime University, Shanghai, where he has been an Associate Professor, since 2017. His research interests include wireless power transfer, control of marine equipment, and prognostics and health management.

Wei Gu received the B.E. and Ph.D. degrees from Shanghai Maritime University, Shanghai, China, in 1982 and 2008, respectively. 
Since 1982, he has been a Teacher with Shanghai Maritime University, where he has been a Professor, since 1997, and is currently the Director of the Key Laboratory of Transport Industry of Marine Technology and Control Engineering. His research interest includes marine information control technology.

\section{Creative Commons Attribution License 4.0 (Attribution 4.0 International, CC BY 4.0)}

This article is published under the terms of the Creative Commons Attribution License 4.0

https://creativecommons.org/licenses/by/4.0/deed.en_US 